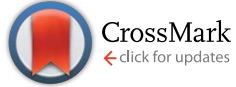

Cite this: RSC Adv., 2015, 5, 20865

Received 17th December 2014

Accepted 4th February 2015

DOI: $10.1039 / c 4 r a 16551 a$

www.rsc.org/advances

\section{Molar ratios of therapeutic water-soluble phenothiazine - water-insoluble phospholipid adducts reveal a Fibonacci correlation and a putative link for structure-activity relationships}

\author{
Hendrik Keyzer, $\dagger^{\mathrm{a}}$ Stephen J. Fey, $t^{\mathrm{b}}$ Barry Thornton ${ }^{\mathrm{c}}$ and Jette E. Kristiansen ${ }^{\star d}$
}

The fact that non-antibiotics can sensitise microorganisms for antibiotic treatment suggests that these molecules have valuable potential to treat multiple drug resistance. Here, we explore the spatial interaction of selected therapeutic phenothiazine hydrochloride derivatives (PTH) with various lipids. Micro-gravimetric titrations were performed on aqueous suspensions of promethazine (PMTZ), promazine (PMZ), triflupromazine (TFM), chlorpromazine (CPZ), prochlorperazine $\cdot \mathrm{HCl}(\mathrm{PCIP} \cdot \mathrm{HCl})$, trifluoperazine $\cdot \mathrm{HCl}(\mathrm{TFP} \cdot \mathrm{HCl})$, prochlorperazine $\cdot 2 \mathrm{HC} 1(\mathrm{PCIP} \cdot 2 \mathrm{HCl})$, trifluoperazine $\cdot 2 \mathrm{HCl}(\mathrm{TFP} \cdot 2 \mathrm{HCl})$ and synthetic 1,2diacyl-sn-glycero-3-phospholipids (PL) with even-numbered symmetric saturated diacyl chains with the headgroups choline (PC), glycerol (PG), serine (PS), ethanolamine (PE), phosphatidic acid (PA). We observed water soluble products with replicable molar ratios (MR) that could be divided into two series. 'Series 1' (PMTZ < PMZ < CPZ < PCIP < TFP) followed the ionization potential and concomitant sedative effects. 'Series 2 ' followed the lipophilicity of $\mathrm{PL}$ and could be related to various non-neuronal physiological aspects. The MR of Series 2 followed the $\mathrm{PL}$ order $\mathrm{PC}<\mathrm{PG}<\mathrm{PS}<\mathrm{PE}<\mathrm{PA}$, echoing the changes in the membrane proportions found in eukaryotic cells proceeding from the plasma membrane to the endoplasmic reticulum. The same order was found when hydrogen ion concentration was increased $\left[\Delta_{\mathrm{i}} \mathrm{H}^{+}\right]$when plotted against the MR for each separate diacyl series. The $\left[\Delta_{\mathrm{i}} \mathrm{H}^{+}\right]$of the PTH/PL was always larger than that of the self-associated pure PTH. In summary, the reproducibility and similarity of the measurements using different PTH derivatives and PL suggests that similar adducts are formed in all the cases. We propose that the PTH.PL adduct has a "helical shape" showing Fibonacci properties for molecules. These observations may open new opportunities for the development of novel therapies.

\section{Introduction}

Chlorpromazine hydrochloride (CPZ) and related exobiotic, amphipathic, micelle-forming phenothiazine (PTH) salts (see Fig. 1A for representatives) have shown therapeutic effectiveness against deleterious micro-organisms and cancer cells. $^{1-6}$ Evidence is mounting that alone or as helper

\footnotetext{
${ }^{a}$ Department of Chemistry and Biochemistry, California State University, 5151 State University Drive, Los Angeles, California 90032, USA

${ }^{b}$ Department of Biochemistry and Molecular Biology, University of Southern Denmark, Campusvej 55,DK-5230 Odense, Denmark.E-mail: sjf@bmb.sdu.dk

'University of Technology, School of Mathematical Sciences, School of Physics, University of Sydney, Sydney, NSW, Australia. E-mail: fthornton@ozemail.com.au

${ }^{d}$ MEMPHYS, Department for Physics, Chemistry and Pharmacy, University of Southern Denmark, Campusvej 55,DK-5230 Odense,Denmark.E-mail: jette-e-k@mail.dk; malthe@dadlnet.dk

$\dagger$ The ideas in this manuscript were pioneered by H. Keyzer. We have completed and submitted the manuscript posthumously in his honour.

\$ These authors contributed equally to the work.
}

compounds, they may revive the use of older, classical antibiotics to combat the alarming rise of multi-resistant microbes world-wide. ${ }^{1,5,7,8}$ PTH, as neuroleptics, involve stereospecific sites, but their mode of action includes so-called "nonneuronal" interactions that may cover many side-effects and contribute to other psychotropic and/or biological activity. ${ }^{9-12}$ Human toxicity, often a concern with PTHs, can be measured in vitro using $3 \mathrm{D}$ cultures, which accurately reflect the in vivo response. ${ }^{13} \mathrm{~A}$ truism exists that the longer a drug is in use, the more side-effects are discovered. After all, micro-organisms have none of the above-mentioned receptors yet become "sedated" or otherwise react to PTH medicinal intervention. ${ }^{14-16}$ Goosey and Doggett pointed out that PTH neuroleptics involve lipophilicity rather than solely shape-specific mechanisms. ${ }^{17}$ The notion that the PTH accumulates in membranes heterogeneously is well-established. ${ }^{18,19}$ Such amphipaths distribute into rich and poor domains in phospholipid (PL) multilayers. ${ }^{20-22}$ For example, similar activity has been noted for alamethacin, which forms micelles in aqueous solutions and 
A

\begin{tabular}{|c|c|c|c|}
\hline \multicolumn{2}{|c|}{ Some PTH molecules } & $R_{1}$ & $R_{2}$ \\
\hline Promazine & PMZ & $\mathrm{H}$ & $-\left(\mathrm{CH}_{2}\right)_{3}-\mathrm{N}\left(\mathrm{CH}_{3}\right) \cdot 2 \mathrm{HCl}$ \\
\hline Chlorpromazine & $\mathrm{CPZ}$ & $\mathrm{Cl}$ & $-\left(\mathrm{CH}_{2}\right)_{3}-\mathrm{N}\left(\mathrm{CH}_{3}\right) \cdot 2 \mathrm{HCl}$ \\
\hline Triflupromazine & TFM & $\mathrm{CF}_{3}$ & $-\left(\mathrm{CH}_{2}\right)_{3}-\mathrm{N}\left(\mathrm{CH}_{3}\right) \cdot 2 \mathrm{HCl}$ \\
\hline Promethazine & PMTZ & $\mathrm{H}$ & $-\left(\mathrm{CH}_{2}\right)_{2}-\left(\mathrm{CH}_{3}\right)-\mathrm{N}\left(\mathrm{CH}_{3}\right) \cdot 2 \mathrm{HCl}$ \\
\hline Trifluoperazine & TFP & $\mathrm{CF}_{3}$ & $\left.-\left(\mathrm{CH}_{2}\right)\right)_{-}-\mathrm{N}=\mathrm{N}^{-}\left(\mathrm{CH}_{3}\right) \cdot 2 \mathrm{HCl}$ \\
\hline Prochlorperazine & PCIP & $\mathrm{Cl}$ & $-\left(\mathrm{CH}_{2}\right)_{3}-\mathrm{N} \smile \mathrm{N}\left(\mathrm{CH}_{3}\right) \cdot 2 \mathrm{HCl}$ \\
\hline
\end{tabular}

B

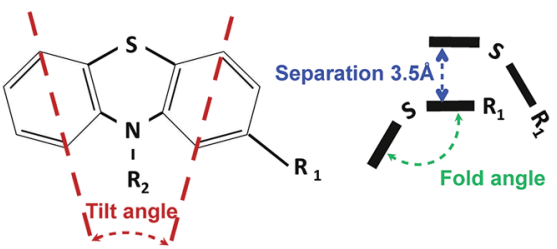

\section{C}

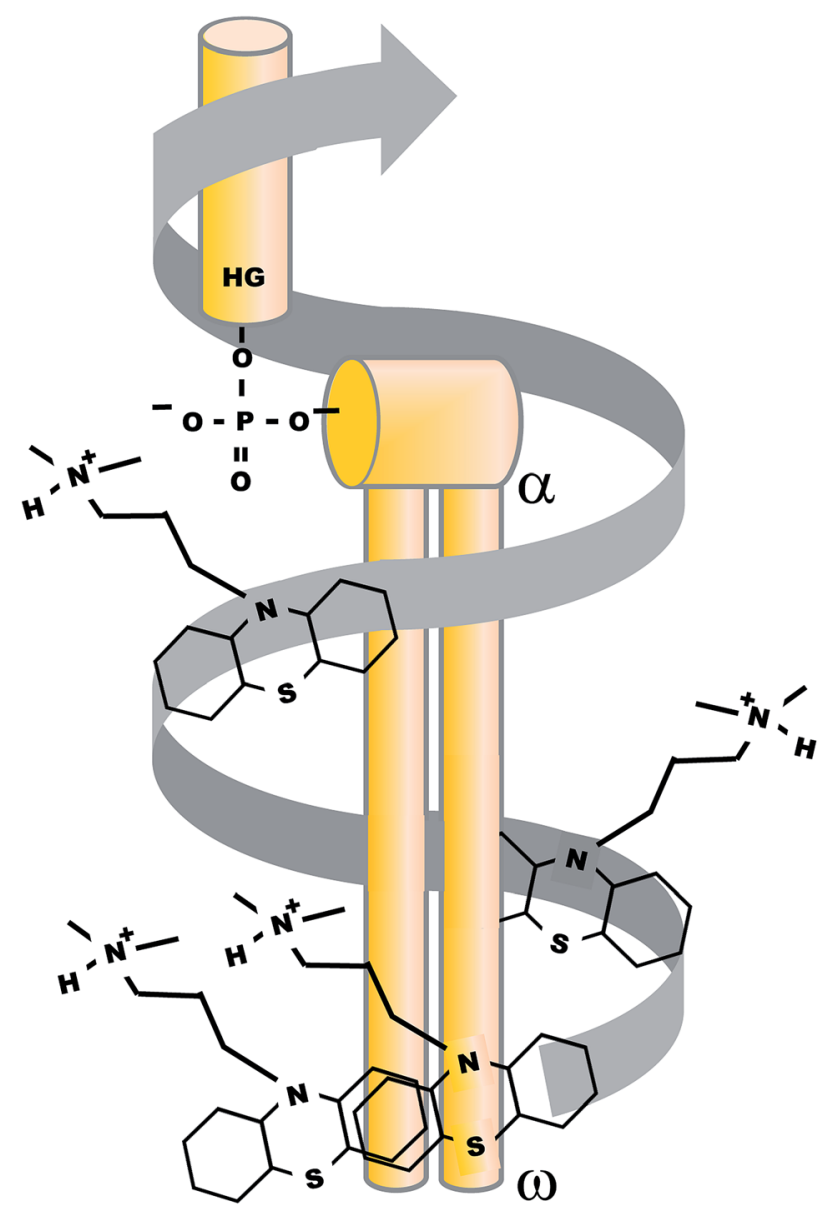

Fig. 1 (A) Therapeutic psychometric phenothiazine hydrochloride derivatives (PTH) used in this study. (B) PTH Geometry. General headgroup structure illustrating the tilt angle induced by the difference in size of the $S$ and $N$ atoms. Diagram of the 'fold angle' between the two benzene rings induced by the $\mathrm{S}$ and $\mathrm{N}$ atoms and the separation distance between the two PTH molecules. (C) Diagram of the proposed helical interaction between the phospholipid (in grey) and the PTH (black). HG $=$ head group, $\alpha-\omega$ the first and last carbon molecules in the acyl tails.

readily incorporates into plasma bilayers, forming channels by self-association. ${ }^{23}$ The critical micelle concentration of $\mathrm{CPZ}$ in water is about $10^{-5} \mathrm{M}^{20}$ With a preference for lipids, cell lysis begins to occur at $3 \times 10^{-5}$ M. ${ }^{22}$ Membrane saturation may be as high as 1 M. $^{20,22}$ Maher and Singer showed that small amphipaths, including CPZ, at concentrations lower than those inducing lysis, promoted gross distribution of components in the plane of quite different membranes, and that this resulted in their highly selective extraction. ${ }^{22} \mathrm{CPZ}$ tends to accumulate in humans mainly in lecithin-rich sites, persisting unchanged for up to 1 year. ${ }^{20,22,24}$ PL are well-known to move in a cellular membrane ${ }^{25,26}$ but the accumulation of PTH at a target PL site, as one would expect from a PTH-enriched domain, may well immobilize the PL (sedate), extract it (inducing lysis), penetrate into, or otherwise disrupt its behaviour in the membrane. Carey and coworkers showed that $\mathrm{CPZ} \cdot \mathrm{HCl}$ solubilized membrane PL efficiently as micellar solutions when the drug/PL molar ratio reached $4: 1 .^{27}$ We found a similar effect with phosphatidylcholine (PC), phosphatidyl glycerol (PG) and an exobiotic, synthetic lipid, discovering in the process, that the molar 
solubilization of PL by PTH was quite reproducible. ${ }^{28}$ Therefore, we examined the effects of various quantities of aqueous PTH in vitro on some major PL groups found in most eukaryotic and prokaryotic membranes. We also examined some putative structure-activity relationships (SAR) other than neurolepticity and propose higher order structures for the PTH/PL interactions.

\section{Results}

The molar ratios (MR) in Table 1 are generally averages of triplicates. All PTH/PL interaction product MRs were replicable to within $\pm 5 \%$. The use of hydrochloride salts was justified: for example, PCLP as the hydrochloride has a PTH/PL MR comparable to that of the edysilate derivative but the maleate derivative yielded an immediate dense precipitate resisting all further additions of PTH to reach an MR. The adducts were generally stable, i.e., did not precipitate until the MR increased for the most part beyond about $40-50$ at which point the initially soluble adducts tended to precipitate after 2 to 24 hours, (precipitation is indicated by the underlined values in Table 1) probably due to splaying of the longer chains. Above the PL 18C, MR endpoint errors generally tended to increase.

Fig. 2 shown a plot with error bars of CPZ/PL MR vs. PL molecular weight (MW). The replicability of the MR data suggested that the PTH/PL products may well be designated as adducts rather than by the looser term aggregates, especially in view of the notion that we may not just be dealing with micelles per se. Advisedly, and henceforth, we eschew the term "micelle" wherever possible. ${ }^{29}$ More evident from Table 1 than from Fig. 2 is that the CPZ/PL adduct MR for comparative $\mathrm{Ci}$ (carbon atoms per chain) always increases in the headgroup order, PC $<$ PG $<$ PS $<$ PE $<$ PA. All PTH/PL adducts exhibited similar profiles. Thus, we believe that similar conformational structures are involved. Table 1 shows the MR of various PTH sequestering PL (C16), and extracts. For the pure PL involved, on the whole, all the PTH/PL MR followed the same trend: PMTZ $>$ PMZ $>$ CPZ $>$ TFM $>$ PClP $>$ TFP, including PI, and

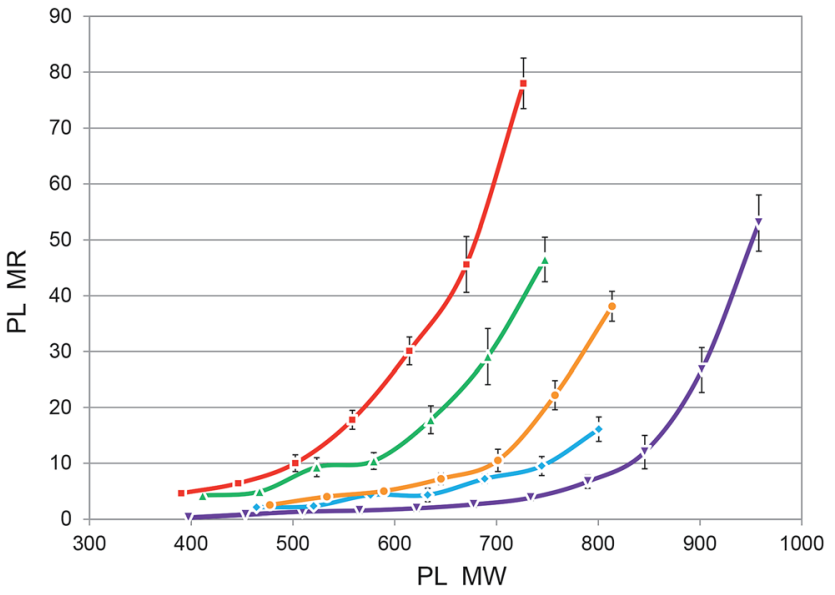

Fig. 2 Plot of the total molecular weight of the phospholipid against the molar ratio PTH/PL for the phospholipids with different chain lengths as given in Table 2 with the following headgroups: $\mathbf{a}$ phosphatidylphosphate (PA); $\Delta$ phosphatidylethanolamine (PE); phosphatidylserine (PS); phosphatidyl glycerol (PG, chain lengths); and $\boldsymbol{\nabla}$ phosphatidylcholine (PC). The range of acyl chain lengths used in all cases was 6-18 except for PC where it was $4-24(n=3)$.

E. coli B. Because the characteristic charge transfer complex adduct colours were observed, especially for the extracts, developing slowly and deepening upon cooling to $0{ }^{\circ} \mathrm{C}$, we concluded that the PTH in their adducts followed excimeric charge transfer complex regimes, as they do in the pure form. ${ }^{20}$ However, charge transfer is confined to the PTH molecules. We found that this phenomenon extended to all the analogues of CPZ in our study, and suggested a universal trait. ${ }^{30}$

\section{Solid state aspects}

Tehrani and coworkers claimed to have observed CPZ nanocrystallites in solution, where the aggregate sizes increased with increasing concentration, confirming the stepwise CPZ aggregation. ${ }^{31}$ Barbosa et al. who used small-angle X-ray scattering and EPR spectroscopy also inferred that $\mathrm{CPZ}$ remained in

Table 1 Molar Ratio (MR) for phenothiazine:lipid extracts. * "Series 1" reads horizontally and "Series 2" reads vertically. ** Extract compositions from Avanti Polar Lipids, Inc. Catalogue VI. Revised. PI: phosphatidylinositol (MW 587): weight\%; 33, $16: 0$ saturated; $46.8,18: 2$ unsaturated. ESM: egg sphingomyelin: area\% FAME-GC/Fl; $16: 0,83.9 ; 18: 0,6.3 ; 20: 0,3.8 ; 24: 0,4.2$. BSM: brain sphignomyelin: "\%": $16: 0,1.7 ; 18: 0$, $45.5 ; 20: 0,5.1 ; 22: 07.2 ; 24: 0,23.3$. Other unsaturated/undetermined. Bold and underlined values indicate that precipitation ensues after $2-$ $24 \mathrm{~h}$ at MR. E. coli B, total extract: weight\%; PE 57.5; PG 16.6; CaL (cardiolipin) 9.8, other 17.6, E. coli B, polar extract: weight\%; PE 67, PG2 3.2, CaL 9.8 , other 0 . Adduct colour of extracts are blue for PMTZ and pink for all the others. Apparent mol. weight of $E$. coli taken arbitrarily as $750 / \mathrm{mol}$ being the rough average of equimolar PE14 and PG18. Other mol. weights as given by Avanti

\begin{tabular}{|c|c|c|c|c|c|c|c|}
\hline MR PTH/lipids* & $\mathrm{PMTZ} \cdot \mathrm{HCl}$ & $\mathrm{PMTZ} \cdot \mathrm{HCl}$ & $\mathrm{CPZ} \cdot \mathrm{HCl}$ & $\mathrm{TFM} \cdot \mathrm{HCl}$ & $\mathrm{PCIP} \cdot 2 \mathrm{HCl}$ & $\mathrm{TFP} \cdot 2 \mathrm{HCl}$ & $\mathrm{TFP} \cdot \mathrm{HCl}$ \\
\hline $16: 0 \mathrm{PC}$ & 38.8 & 22.4 & 3.8 & 20.9 & 11.6 & 27.7 & 22.5 \\
\hline $16: 0 \mathrm{PG}$ & 49 & 29.1 & 9.5 & 25.8 & 30.6 & 56.5 & \\
\hline $16: 0 \mathrm{PE}$ & 76 & 61 & 29.1 & 77 & & $\overline{112}$ & \\
\hline $16: 0 \mathrm{PA}$ & 169 & & 46.6 & $3 \overline{17}$ & & $\overline{143}$ & \\
\hline $\mathrm{PI}^{* *}$ & 49 & 37 & 13 & $\overline{16}$ & & $\overline{18}$ & \\
\hline E. $\operatorname{coli}$ B total ${ }^{* *}$ & 127 & 86 & 22 & 87 & & 145 & \\
\hline E. coli B polar** & 88 & 83 & 18 & 35 & & 129 & \\
\hline
\end{tabular}


solution as nanocrystallites. ${ }^{32}$ We believe that for PTH and analogues to aggregate in solution stepwise implies heterocycle to heterocycle interactions, i.e. by excimerization, as confirmed by Mukerjee and Gosh, for the self-association of methylene blue into multimers ${ }^{33}$ (Fig. 1B). PTH side chains serve to stabilize aggregation via solvent enforcement. ${ }^{31}$ At the nanostructural levels, CPZ in solution answers to the demands of solid state characteristics. ${ }^{20,32,34}$

\section{Impurity centres}

In aggregates of PTH, intermolecular charge transfer complexing is a way of aiding electron delocalization, just as the addition of a foreign molecule as an impurity centre does. ${ }^{20,34,35} \mathrm{We}$ regard the PL as such an impurity centre. An aggregate structure of this kind makes a considerable contribution to the free energy of the system. At $300 \mathrm{~K}$, this free energy gain is about $0.055 \log N \mathrm{eV}$. In a monolayer of just 20 molecules, the energy gain is quite substantial, about $0.072 \mathrm{eV}$. $c f$., the approximate hydrogen bond value of $0.08 \mathrm{eV}$ in bulk water. ${ }^{20}$ The clearest feature of an excimeric charge transfer complex is the reversibility of equilibrium with its isolated components, according to the reaction scheme (second and third step) in eqn (1) (from (ref. 20)):

$$
\begin{aligned}
& \mathrm{D} \text { (donor) }+\mathrm{A} \text { (acceptor) } \Leftrightarrow \mathrm{DA} \text { (contact } \mathrm{CT}) \Leftrightarrow \\
& \mathrm{DA}^{*}(\text { exiplex or excimer }) \Leftrightarrow \mathrm{D}^{+}-\mathrm{A}^{-} \Leftrightarrow \mathrm{D}^{+}+\mathrm{A}^{-}
\end{aligned}
$$

for an excimer $\mathrm{D}^{*}=\mathrm{A}^{*}$.

The energy differences involved in such excitation processes are generally small, in the order of $k T$, and can therefore be produced by a variety of supplying mechanisms, (including electrochemical phenomena at membrane surfaces), ${ }^{36,37}$ with profound consequences for electron transfer processes in the presence of PTH..$^{20}$ For high stability, charge transfer should be incomplete. ${ }^{38}$ Excimeric electron affinity is increased if the electron is taken not to the bottom of the conduction band but into an excited state energy level leading to enhanced complexation. If the electron is raised not from the Fermi level but from a higher excited state energy level, the ionization potential is reduced leading again to enhanced complexation. Both processes can occur simultaneously. Excimeric charge transfer complexes play important roles in many biological processes. ${ }^{20}$ A corollary of aqueous assemblages of such particles is that counter-ions and water molecules are attracted by the hydrophilic regions, and assume extensively ordered states. Excitation also leads to considerable changes in magnitude and direction of the dipole moments. ${ }^{39,40}$

\section{pH effects}

Self-association of PTH reduces the $\mathrm{pH}$ of the cationic drug assembly, such as that seen for PMZ, CPZ and thioridazine (TDZ). ${ }^{29,41,42}$ Furthermore, all PTH/PL adducts were more acidic in PTH concentrations equivalent to the pure drugs. The straight line plots in Fig. 3, in which the hydrogen ion concentration differences $\left(\left[\Delta_{\mathrm{i}} \mathrm{H}^{+}\right]\right)$vs. MR show a regular increase, is evidence that the PTH/PL adducts have specific, and

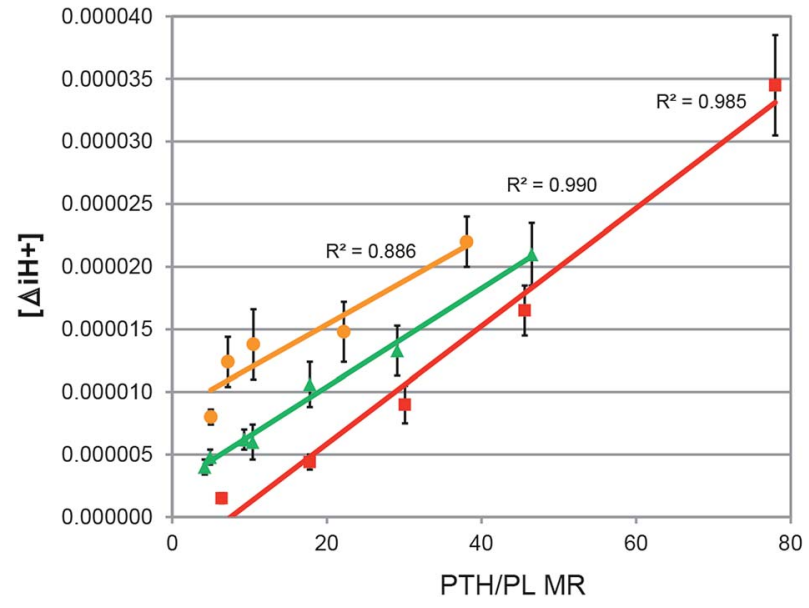

Fig. 3 Plot of the differences in the hydrogen ion concentration $\left(\left[\Delta_{\mathrm{i}} \mathrm{H}^{+}\right]\right)$ between pure PTH and the PTH/PL adduct plotted against the molar ratios (MR) of three different classes of $\mathrm{PTH}$, where the classes have the same head group but different phospholipid chain lengths (PL) $\square$ C18 phosphatidylphosphate PA, $R^{2}=0.97 ; \Delta$ C16 phosphatidylethanolamine PE, $R^{2}=0.99$; $C 14$ phosphatidylserine $\mathrm{PS}, R^{2}=0.92(n=3)$.

similar, structures (definition: $\left[\Delta_{\mathrm{i}} \mathrm{H}^{+}\right]=\left[\mathrm{H}^{+}\right]_{\mathrm{PTH} / \mathrm{PL}}-\left[\mathrm{H}^{+}\right]_{\text {PTHpure }}$ at equimolar PTH concentrations). Within the cohorts of PL, with different headgroup but the same $\mathrm{Ci}$, the plots for the smaller $\mathrm{Ci}$ show concomitant smaller $\left[\Delta_{\mathrm{i}} \mathrm{H}^{+}\right]$increases, as exemplified in Fig. 3. This figure shows that $\left[\Delta_{\mathrm{i}} \mathrm{H}^{+}\right]$is strongly dependent on the acyl chains. The $\left[\Delta_{\mathrm{i}} \mathrm{H}^{+}\right]$increase with increasing MR indicates that the protonated side-chains of the adduct PTH molecules orient away from the hydrophobic PTH/ PL core, and thus structure the immediately adjacent (vicinal) water environment and by extension, lower the $\mathrm{pH}$ of the bulk solution. Water-assisted aggregation forces the PTH molecules into higher-order ion clusters, and increases the dielectric environment; hence, forcing the proximity of the heterocyclic ring donor and ring acceptor groups. This is expected to strengthen charge transfer excimerization..$^{20}$ The PL acyl chains are then also forced into closer proximity by the hydrophobic PTH ring assemblies. The plots indicated that the different PL headgroup interacted with the PTH in a closely similar manner, irrespective of whether the PL were zwitterionic or salts. Note that the points on each line in Fig. 3 again follow the PL MR hierarchy, as shown in Fig. 2 ( $\mathrm{PS}>\mathrm{PE}>\mathrm{PA}$ ).

\section{Influence of the headgroup}

Yeagle claimed that the PL headgroup and acyl chains may be treated as being partially independent of motion and may thus be treated separately. ${ }^{43}$ In terms of imbibing water, i.e., water binding, eggPE < eggPS < eggPC, follows the hierarchy of CPZ/ PL MR vs. PL MW ${ }^{44,45}$ (Fig. 2). A single methyl group added to PE (PE Me) sees a $28 \%$ increase in hydration while successive methylations increase the hydration by $7 \%$ and $16 \%$, respectively. ${ }^{46,47}$ Such hydration increases are also seen for successive methylations of dioctanoyl-PE and dimyristoyl-PE. ${ }^{48-50}$ We thus infer that full PL hydration is observed in all our experiments. The solubility of the PL as a function of the headgroup plays a 
major part in the MR hierarchy shown in Fig. 2. PC is the most soluble of the groups in Fig. 2 and phosphatidic acid (PA) the least, reflected in increasing $\mathrm{MR}$ according to the adducts with the same diacyl chains in the order $\mathrm{PC}<\mathrm{PG}<\mathrm{PS}<\mathrm{PE}<\mathrm{PA}$. Not only is solubility a factor but the adducts of PL with larger headgroup volume have smaller $\left[\Delta_{\mathrm{i}} \mathrm{H}^{+}\right]$(Fig. 3). All the PTH/PL interactions exhibit similar $\left[\Delta_{\mathrm{i}} \mathrm{H}^{+}\right]$changes. This suggests that the initial site of adduct formation is situated nearer the $\alpha$ part of the diacyl chains than the headgroup (Fig. 1C). A larger dynamic headgroup as well would provide more steric hindrance to the initial PTH anchoring site. This is seen for the series in Fig. 4, in which the headgroup size increases by virtue of methyl additions 16PE $<16$ PENMe $<16$ PEN2Me $<16 \mathrm{PC}$ whose MR fall on the 16PL line in Fig. 3, and concomitantly exhibit smaller $\left[\Delta_{\mathrm{i}} \mathrm{H}^{+}\right]$in that order.

\section{Influence of the diacyl chains}

Fig. 2 and 3 show unambiguously that longer diacyl chains elicit larger PTH/PL MR. Considering the steric hindrance of the headgroup, the MR for PTH/PL is an index of the lipophilicity of the PL in question. Thus, for example, PA, the most lipophilic of the PL in our experiments, must acquire a larger quantity of PTH for its adducts to become water-soluble, as indeed is observed. This phenomenon may be explained by placing the lipophilic portion of the PTH nearer to the initial anchoring site of PA namely at $\alpha$ in its diacyl chains (Fig. 1C). Further, to render the adduct soluble, more PTH has to be marshalled to that site. In turn, as the length of the diacyl chains is increased, the PTH excimeric ribbon must be extended to cope with the increasing lipophilicity of the PL until a point is reached where the splay and motion of the diacyl chains overcomes the cohesive excimeric and the vicinal water structure energetic regimes that keep the adducts soluble. This usually occurs when an MR of about 40 to 50 is reached. This view explains why the adduct of CPZ/24PC (MR 53) CPZ/18PS (MR 38) and CPZ/16PE (MR 46) precipitate overnight, while CPZ/18PA (MR 78) precipitates within several hours (see the bold MR values in Tables $2 \mathrm{~A}$ and $2 \mathrm{~B}$ ).

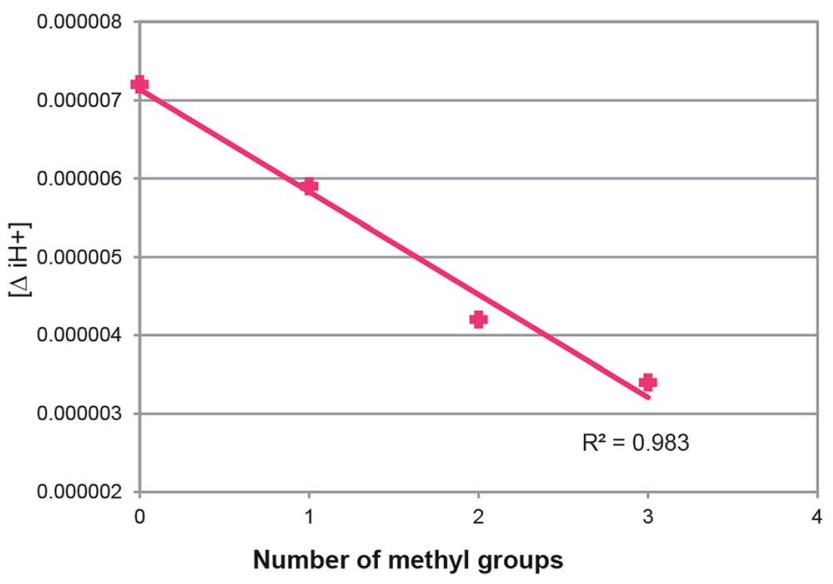

Fig. 4 Plot of the differences in the hydrogen ion concentration $\left(\left[\Delta_{\mathrm{i}} \mathrm{H}^{+}\right]\right)$versus the number of methyl groups for $\mathrm{C} 16$ phosphatidylcholine; $R^{2}=0.98(n=3)$.
Table 2 (A) MR of PTZ/PL combinations and (B) their differences with increasing acyl chain length. Bold and underlined values indicate that precipitation ensues after 2-24 h at MR. Note that equal differences tend to fall on a diagonal (see for example the values in bold type)

\begin{tabular}{|c|c|c|c|c|c|}
\hline \multirow{2}{*}{$\begin{array}{l}\mathrm{A} \\
\mathrm{CL} / \mathrm{MR}\end{array}$} & \multicolumn{5}{|c|}{ Molar ratio (MR) } \\
\hline & $\mathrm{PC}$ & PG & PS & $\mathrm{PE}$ & PA \\
\hline 4 & 0.27 & & & & \\
\hline 6 & 0.8 & 2.1 & 2.5 & 4.2 & 4.6 \\
\hline 8 & 1.3 & 2.3 & 4 & 4.9 & 6.4 \\
\hline 10 & 1.5 & 4.3 & 5 & 9.3 & 10 \\
\hline 12 & 1.9 & 4.3 & 7.2 & 10.4 & 17.8 \\
\hline 14 & 2.6 & 7.2 & 10.5 & 17.8 & 30.1 \\
\hline 16 & 3.8 & 9.5 & 22.2 & 29.1 & 45.6 \\
\hline 18 & 6.7 & 16.1 & 38.1 & 46.5 & 78 \\
\hline 20 & 12 & & & & \\
\hline 22 & 26.7 & & & & \\
\hline 24 & 53 & & & & \\
\hline 16PE-NMe & & & & 14.8 & \\
\hline 16PE-NMe2 & & & & 5.5 & \\
\hline
\end{tabular}

B

Molar ratio differences with increasing acyl chain length $(\Delta \mathrm{MR})$

\begin{tabular}{lllllll} 
CL/MR & CL (average) & PC & PG & PS & PE & PA \\
\hline $6-4$ & 5 & 0.53 & & & & \\
$8-6$ & 7 & 0.5 & 0.2 & 1.5 & 0.7 & 1.8 \\
$10-8$ & 9 & 0.2 & 2 & 1 & 4.4 & 3.6 \\
$12-10$ & 11 & 0.4 & 0 & 2.2 & 1.1 & $\underline{7.8}$ \\
$14-12$ & 13 & 0.7 & 2.9 & 3.3 & $\underline{7.4}$ & 12.3 \\
$16-14$ & 15 & 1.2 & 2.3 & $\underline{\mathbf{1 1 . 7}}$ & $\mathbf{1 1 . 3}$ & 15.5 \\
$18-16$ & 17 & 2.9 & $\underline{6.6}$ & 15.9 & 17.4 & 32.4 \\
$20-18$ & 19 & $\underline{5.3}$ & & & & \\
$22-20$ & 21 & $\mathbf{1 4 . 7}$ & & & & \\
$24-22$ & 23 & 26.3 & & & & \\
Partial sum & $\Delta$ MR for & 5.9 & 14 & 35.6 & 42.3 & 73.4 \\
$(8-18)$ & & & & & &
\end{tabular}

\section{Sedative effects}

Some workers claimed that the sedative effect of clinical doses of psychotropes for stereospecific site interaction increased in the order, $\mathrm{PMTZ}<\mathrm{PMZ}<\mathrm{CPZ}<\mathrm{PClP}<\mathrm{TFP}$ ("Series 1 " first row in Table 1). ${ }^{51,52}$ The PTH charge transfer complex order depends on the electron withdrawing power of the substituent in the heterocyclic 2-position, $-\mathrm{CF}_{3}>-\mathrm{Cl}>-\mathrm{H}$, with the consequent increasing ionization potential $\left(I_{\mathrm{p}}\right)^{53,54}$ as well as flexibility of the molecules. ${ }^{52}$ Fulton and Lyons suggested that the increasingly sedative effect of PTHs tended to follow their decreasing ionization potential $I_{\mathrm{p}}{ }^{47}$ However, they emphasised caution in this respect, because there was little difference between the tranquilizers and stimulants. A smaller $I_{\mathrm{p}}$ allows the easier loss of an electron in a charge transfer event. However, it is necessary to consider that charge transfer in an excimer context is a probability process, in which the electron may not actually be lost by the donor but may spend more time near the acceptor. Thus, physiological activity does not depend on the electron donor power alone. Conformational factors and the lipophilicityinfluencing activity of psychomimetic drugs has been discussed extensively. ${ }^{\mathbf{9}, 55-58}$ 


\section{Non-neuronal effects}

Our MR data is at odds with the sedative clinical information above and may indicate non-neuronal aspects. The PTH aromatic rings are capable only of butterfly wing motion about the $\mathrm{N}-\mathrm{S}$ axis, rarely if ever inverting, whereas the side-chains are flexible depending on the length and substituents. When conformational factors of the PTH in its PL adducts are examined, their correlation with the MR becomes evident. This is $\mathrm{PC}<\mathrm{PG}<\mathrm{PS}<\mathrm{PE}<\mathrm{PA}$ and referred to as "Series 2" (Table 1, first column). While Series 2 is concerned with the PTH/PL adducts only, this leads to a possible insight into other clinical effects. PMTZ has a methyl substituted in the two-carbon side-chain and will have a less flexible side-chain. PMZ, CPZ and TFM share a simple propylamine side-chain. While the strength of the electron withdrawing groups in the ring 2-position plays an important role, the sizes of those groups also increase in the order, $\mathrm{H}<\mathrm{Cl}<\mathrm{CF}_{3}$, inducing a steric hindrance effect that may affect excimerization. The perazines have a piperazinyl sidechain that is longer than the simple propylamine group. Moreover, the piperazinyl group introduces two quatemizable $\mathrm{N}$ atoms, leading to boat or chair conformations depending on whether one or both are protonated..$^{58}$ The perazine boat form $\left(\right.$ mono- $\left.\mathrm{H}^{+}\right)$is preferred at a $\mathrm{pH}$ of approximately 5 , i.e., within the conventional clinical range of $\mathrm{PTH}$ with a propylamine sidechain. The di-protonated piperazinyl chair form exists in the pH range of about $3 .^{58}$ Table 1 shows that TFP.HCI (boat form) has a considerably lower MR than the chair form $\mathrm{TFP} \cdot 2 \mathrm{HCl}$ for 16PC, indeed invariably so for all TFP/PC adducts with corresponding Ci tested. There is no doubt that this phenomenon involves vicinal water, which is less structured for the chair than the boat form. We suggest that the psychomimetic effect follows Series 1 until the available stereo-specific sites are occupied or even saturated, after which the unused excess PTH are exposed to $\mathrm{PL}$ in non-neuronal terms (i.e. in "Series 2" order Table 1: PC $>$ PG $>$ PS $>$ PE > PA). Thus the process for self-associated PTH accumulation at membranes has several avenues available for interaction. The tertiary PTH interaction is mainly at the intrinsic proteins; quatemized PTH interacts only with phospholipids. ${ }^{59}$ The relatively distinct sets of physiological action can now be ascribed to one series (Series 1) involved with neurolepticity requiring a specific conformation of a single PTH at a stereoselective protein site, and to another (Series 2) with a much more generalized interaction of a PTH pseudopolymer at the available membrane PL sites. The overlap of Series 1 and 2 is yet to be determined.

\section{Putative PTH/PL structure}

The conventional view of amphipathic PTH aggregates being spheroidal, ellipsoidal, cylindrical or disc-shaped may not apply in our case. ${ }^{60}$ A recent study of the self-assembly of PTH compounds was inconclusive with respect to the assignment of three-dimensional models: ellipsoids, cylinders or parallelepipeds. ${ }^{32}$ Although at low concentrations, the aggregate structure may approximate the Hartley spherical model, this is unlikely to prevail in more concentrated solutions, as confirmed by streaming double refraction and X-ray diffraction measurements. ${ }^{32}$ In the mixed PTH/PL systems, different three-dimensional criteria are likely to come into play, as has been observed with aggregates of PL and other ionic and non-ionic surfactants. See for example the work of Almgren, who observed lace-like or thread-like structures for $\mathrm{CPZ}$ without intermediates in the form of disks. ${ }^{61}$ Zuchowski and Durand showed that cationic PTH amphipaths interact with artificial and biological membranes so that after the amphipath enters the membrane, a new type of interaction occurred. ${ }^{62}$ Zhao's group, working with paclitaxel (an anticancer compound with an ionisable tertiary $\mathrm{N}$ group), showed that incorporation of the drug into PL models differed according to the headgroups, alky 1 chain lengths and unsaturation. ${ }^{63-65}$

Recently, Kopec and Khandelia carried out some computerassisted predictions of the interactions between the antituberculosis candidate drug thioridazine and two different model bilayers a zwitterionic POPC (1-palmitoyl-2-oleoyl-snglycero-3-phosphocholine) and a negatively charged, mixed POPC/ POPS (1-palmitoyl-2-oleoyl-sn-glycero-3-phospho-L-serine). ${ }^{66}$ They have made a number of predictions that were coherent with the ideas presented here. Firstly, they proposed that the positively charged protonated amino group of THZ's piperidine ring interacts with both the lipid headgroup and the glycerol backbone region via electrostatic interactions (salt bridges) and/or hydrogen bonds similar to that illustrated in Fig. 1C. Secondly, they predicted that the preferential orientation of the molecule with the phenothiazine ring was placed almost perpendicularly to the bilayer, as seen in Fig. 1C. They also noted that this is the preferred orientation of cholesterol. ${ }^{67}$ Finally, they reported that the strong interaction of the piperidine ring with the lipid phosphate group and glycerol backbone, will limit the conformational possibilities of the aromatic tricycle, making a parallel alignment highly unfavourable. This is in agreement with the hypothesis proposed here, where the aromatic tricyclic rings are only partially overlapped. This, the fold and the tilt angle (Fig. 1B) will provide separation in three dimensions for the piperidine ring.

\section{Phospholipid splay angles}

If one considers for simplicity the PTH to be arranged in circular rings, then by calculating the difference between the MR for increasing $\mathrm{Ci}$ steps, then one can obtain a rough estimate of the number of CPZ molecules that bind to the additional chain length of the PL (Table 3). Knowing this, one can calculate the fold angle around the S-N axis (Fig. 1B).

In geometric algebra terms, the sequence of numbers in Table 3 can be considered a source for providing progressive values of $2 \mathrm{D}$ orthogonal projections of molecular locations on the spiral pathway onto a flat surface, the values of which determine the splay angle and pitch of the spiral ( $c f$. an inverse problem in mathematics or time consuming analysis).

Assuming the CPZ excimer to be fully overlapped then the length $Q$ (Fig. 1B) can be used to calculate the effective length of the sides of the polygon descried by the PTH molecules. The absolute length for $Q$ varies from about 2.75 to $3.25 \AA$ depending on which source is used (CSR space filling or ChemDraw models 
흔 흔

幽

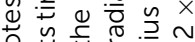

乙先范

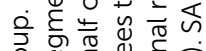

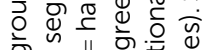

व)

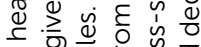

है

品 $ᄃ$ O

迹

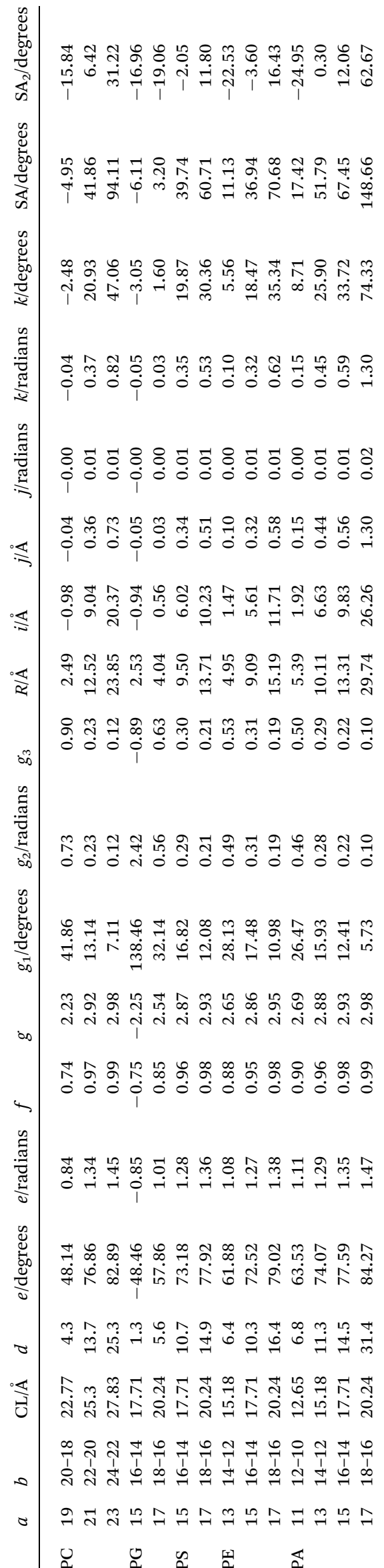

of various PTH derivatives). Its absolute length will be reduced to an effective length because of the fold and the thickness of the carbon ring. An absolute length of $3 \AA$ is used for the calculations presented in Table 3 (although other values in the 2.75-3.25 range do not result in significantly different conclusions). Once the length of the polygon edge formed by the CPZ is known, it is possible to calculate the radius of the inscribed circle. Yeagle (Chapter 1, Tables 1-7) gave the cross-sectional areas of several different phospholipid chains, which can be used to provide an averaged area of $38.05 \AA^{2}{ }^{23}$ Using the radius of the phospholipid chain thus to be $3.48 \AA$, it is then possible to calculate the splay angle of the phospholipid chains. Once again it is possible to see that the Series 2 order is maintained $(\mathrm{PC}<\mathrm{PE}<\mathrm{PA})$ when the splay angle is compared with the chain length (Fig. 5).

Returning to the spiral concept presented in Fig. 1C, it would appear that at longer chain lengths, due to the splay angle of the phospholipid chains, that the spiral is in fact some sort of helix (Fig. 6). This could be anticipated considering the tilt and fold angles of the PTH molecule. From the available data, it is not possible for us to decide whether the helix has one or more threads around the central phospholipid chains and so Table 3 gives the splay angles for a single or two threads as examples.

One well-known series of numbers, often associated with a spiral in nature, is the Fibonacci series $(1,1,2,3,5,8,13,21,34$, $55 \ldots)$ as seen in the seeds on a sunflower, the spirals of shells, and the curve of waves. In theoretical studies in the mathematics of fractals for constructions using triangles (in 2D), the results show that the Fibonacci sequences can be found in the gaps formed.$^{68}$ Here it is interesting to note that if the molar ratios determined here for all five PTH (PC, PG, PS, PE and PA) are plotted against this series, then one obtains straight line graphs with a correlation coefficient greater than 0.987 in all cases (Fig. 7). Its significance was unclear until it was recognised as being related to energy optimisation in $3 \mathrm{D}$, such as in some nature examples (e.g. spinal growth in some plant locations along a stem), However, this is one of the first observations of the Fibonacci series fitting at the molecular level.

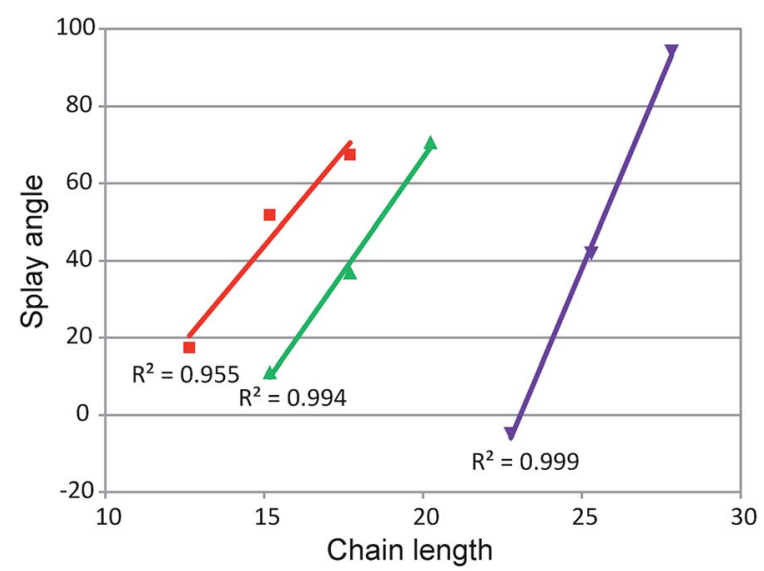

Fig. 5 Plot of the splay angle versus the chain length for $\mathbf{\square}$ phosphatidylphosphate (PA, $\left.R^{2}=0.96\right) ; \boldsymbol{\Delta}$ phosphatidylethanolamine $\left(\mathrm{PE}, R^{2}=0.99\right)$; and $\boldsymbol{\nabla}$ phosphatidylcholine (PC, $\left.R^{2}=0.99\right)(n=3)$ calculated as shown in Table 3 


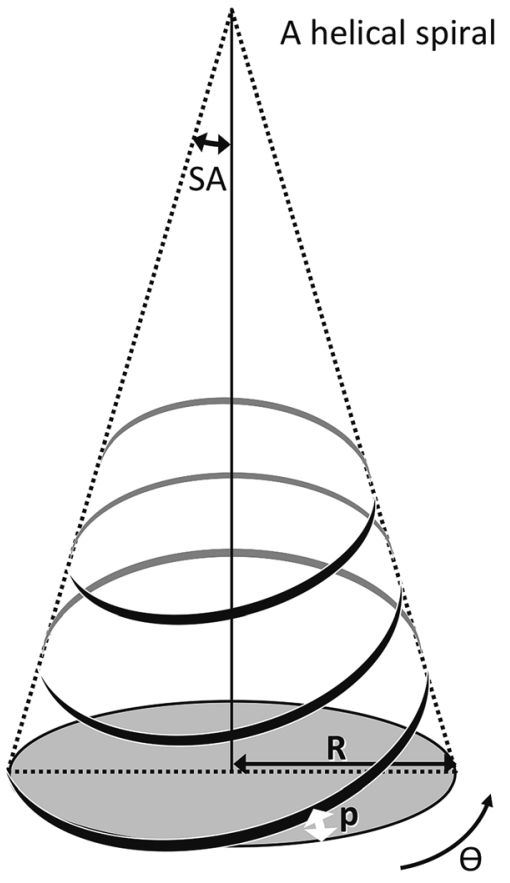

Fig. 6 The key definitions for a helix. $\alpha=$ splay angle; $p=$ pitch; $R=$ radius and $\theta$ circumferential angle.

\section{Correspondence of PTH/PL and PL}

In terms of cellular biosynthetic routes (precursor $\rightarrow$ product): $\mathrm{PA} \rightarrow \mathrm{PG}, \mathrm{PA} \rightarrow \mathrm{PI}, \mathrm{PA} \rightarrow \mathrm{PS} \rightarrow \mathrm{PE} \rightarrow$ PENMe $\rightarrow$ PEN2Me $\rightarrow$ $\mathrm{PC} \rightarrow \mathrm{SM}^{69-71}$ it is remarkable that product $\mathrm{PTH} / \mathrm{PL} \mathrm{MR}$ is almost always smaller than that of the precursor PTH/PL MR (the exception being PS $\rightarrow$ PE); see Table 1 and Fig. 2. The MR indicates that fewer PTH molecules are used to enclose (and modify the properties of) PA than PS.
It has long been appreciated that lipid species are not distributed equally among the various cellular membranes ${ }^{72,73}$ and that the membrane itself is asymmetric and has substructures including rafts. ${ }^{74}$ In the outer leaflet in bacteria, after closely bound PE and cardiolipin (CL), the third major PL is PG. van Meer, Voelker and Feigensohn produced an elegant article on the location, prevalence and distribution of PL within a eukaryotic cell. ${ }^{74}$ In terms of concentration and traversing the cell from the outer cell membrane to the 'core' (endoplasmic reticulum and mitochondria) of the cytosol, the PL distribution tends to follow the Series 2 order, i.e. $\mathrm{PC}>\mathrm{PG}>\mathrm{PS}>\mathrm{PE}>\mathrm{PA}^{22,74}$ Interestingly, PE, and PS are located preferentially on the cytosolic side of the plasma membrane, whereas sphingomyelin (SM) is located predominantly in the outer layer (luminal side) of the plasma membrane. This gradation results in inner membranes being loosely packed while the outer membranes are stiffer and closer to adopting a 'solid' gel phase.

Therefore, the PL most protected from external assault (treatment) by the PTH in eukaryotic cells is PA, which is also the precursor of ultimately all the PL products mentioned above. The next are PE, and PS. Thus, the most PTH 'sensitive' PL are the PL that are the most protected and the least sensitive are the most exposed. This may well have important consequences for PTH treatments.

Tumour cells produce extra PG and PC, and this apparently contributes to both proliferative growth and programmed cell death mechanisms. ${ }^{75} \mathrm{PC}$, sphingomyelin (SM) and PG have also been found in higher proportions in various cancer cells. ${ }^{76}$ This may be present convenient targets for interaction with PTH.

Children with frequently recurring pyelonephritis responded better to combined PMTZ/antibiotic treatments than to antibiotics alone; and no new renal scarring was observed in the PMTZ-tested group. ${ }^{20}$ Free floating $E$. coli are likely destroyed

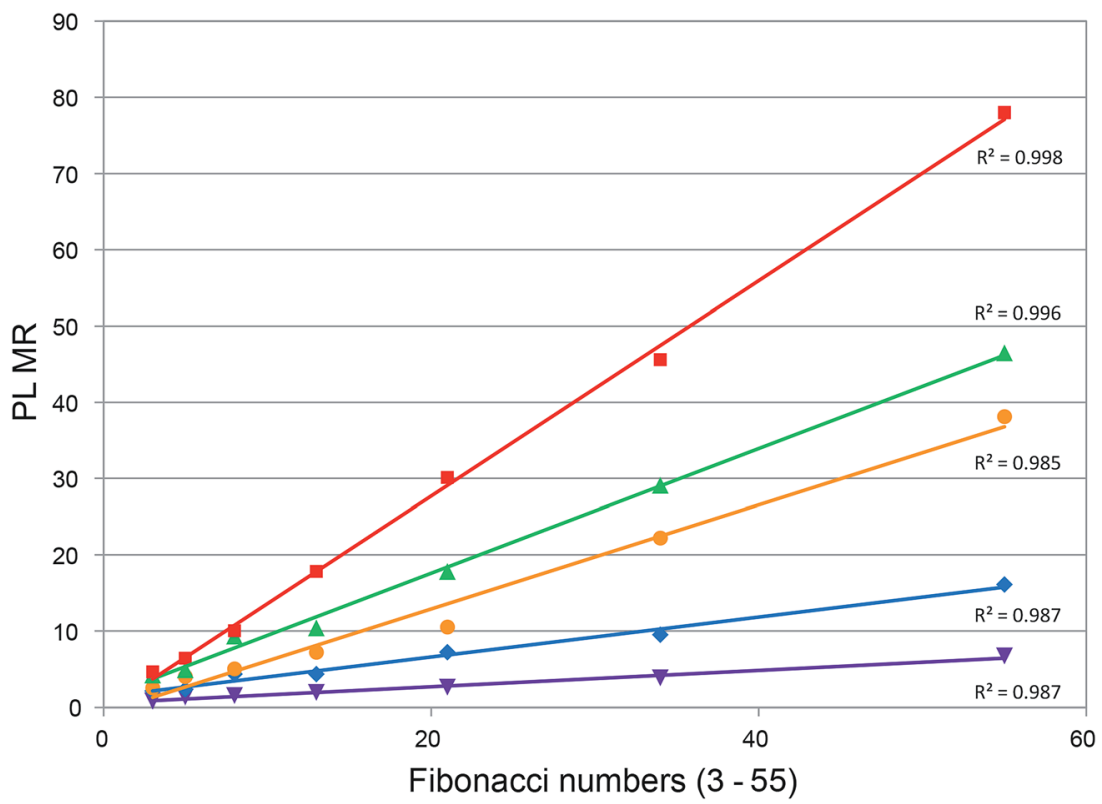

Fig. 7 Plot of the molar ratio of PTH/PL for the phospholipids with different chain lengths against the Fibonacci numbers from 3 to 55 . Headgroups: $\mathbf{D}$ phosphatidylphosphate (PA, $\left.R^{2}=0.99\right) ; \boldsymbol{\Delta}$ phosphatidylethanolamine (PE, $\left.R^{2}=0.99\right) ; 0$ phosphatidylserine (PS, $\left.R^{2}=0.98\right) ;$ phosphatidyl glycerol (PG, $\left.R^{2}=0.98\right)$; and $\boldsymbol{\nabla}$ phosphatidylcholine (PC, $\left.R^{2}=0.98\right)$. The range of acyl chain lengths used in all cases was $6-18$. 

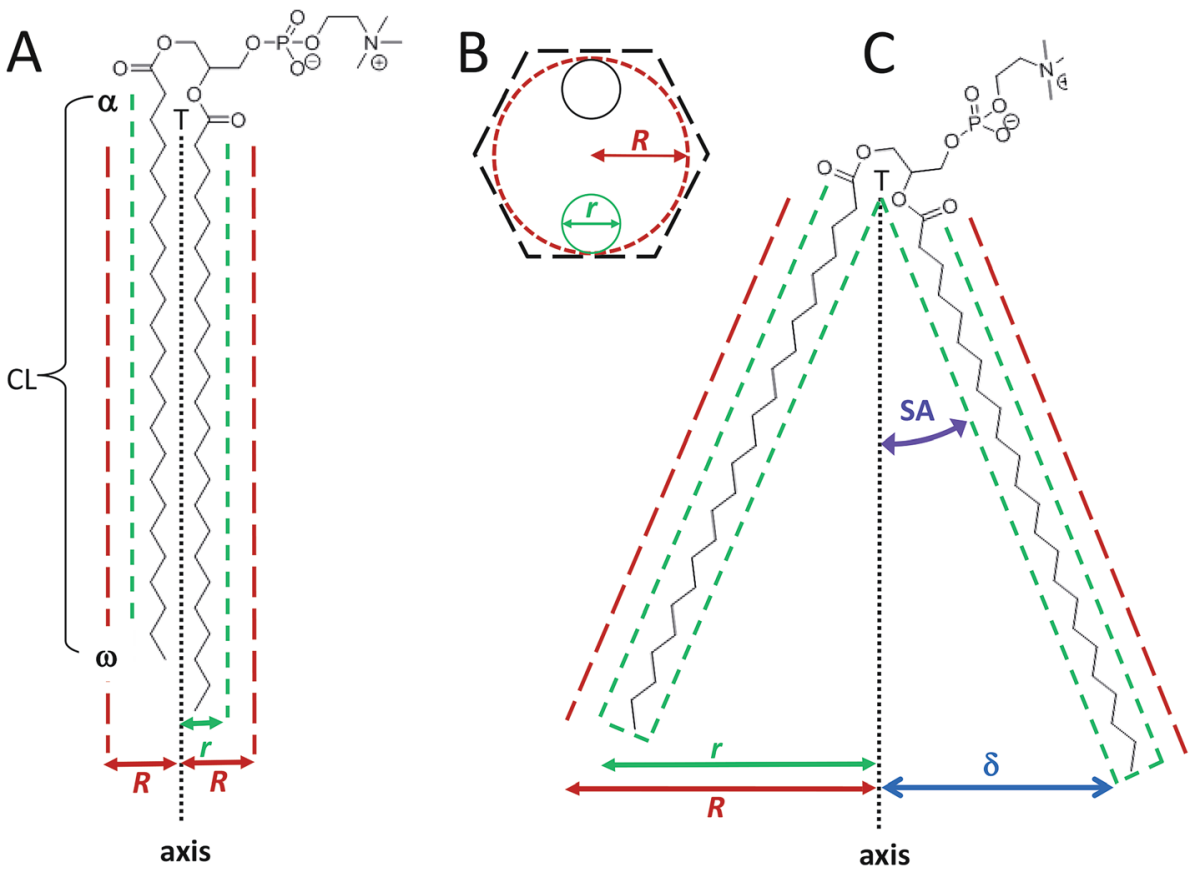

Fig. 8 Diagram of the key measurements for the splayed phospholipid. (A) Line T indicates the mid-axis between the two acyl chains of the phospholipid of chain length $\mathrm{CL}$ (from $\alpha$ to $\omega$ ). $r=$ the circumferential diameter around one acyl chain. $R=$ the radius of the bounding circle of the polygon. (B) Diagram of the relationship among $r, R$, the polygon and its bounding circle. The polygon and bounding circle are separated for clarity. (C) Splayed phospholipid. SA is the splay angle and $\delta$ is the difference $2 R-2 r$.

first, while the adherent pathogens are more tenacious because less surface area is exposed, PTH/PG still being involved.

\section{Discussion}

If the PTH forms spiral metastructures around phospholipids, what would be the effects and consequences?

Considering the PTH molecule itself, the hydrophobic ring structures are reasonably flat and thus would presumably tolerate a reasonable amount of relative rotational movement. This would allow the variable pitch in the spiral needed for different splay angles (SA and $p$ in Fig. 7 respectively).

While one would anticipate that the fold angle (Fig. 1B) would be quite inflexible, the effective fold angle may actually be augmented by allowing the hydrophobic ring structures to rock on each other. This is probably necessary to achieve the range in number of PTH molecules in the rings/spirals around the phospholipid (as given in Table 3 column ' $d$ ').

The resolution of the molar ratios into straight lines by plotting them against the Fibonacci series is especially intriguing. This is interesting regarding Fibonacci numbers and the coverage of special sections. The generating function $f(x)$ of the Fibonacci numbers satisfies the identity:

$$
\left(1-x-x^{2}\right) f(x)=x
$$

which leads to the closed form

$$
t^{n}-\left(1-t^{n}\right) / \sqrt{ } 5
$$

where

$$
t=(1+\sqrt{ } 5) / 2
$$

This is the so-called 'golden-mean' (or 'golden ratio') of identical copies of a fundamental structural unit cell oriented in the same way. This again suggests a highly ordered alignment of the PTH molecules around a central point.

The splay of the phospholipid itself induced by the PTH molecule would lead to a local membrane thinning and changes in membrane fluidity and functionality. Membrane thinning and expansion has been observed in other biological situations, including binding to antimicrobial peptides and to PTH. ${ }^{66}$ Antimicrobial peptides have been shown to produce perturbations of the phospholipid membrane by the formation of specific lipid-peptide domains, lateral phase segregation of zwitterionic from anionic phospholipids and even non-lamellar lipid phases. ${ }^{77}$ Furthermore, some peptides change their topology in a $\mathrm{pH}$ dependent manner and these peptides have been shown to be more potent in antibiotic assays under experimental conditions, where they show approximately in-plane alignments. ${ }^{78}$ In an interesting study using four of the phospholipids used here (PC, PG, PS and PE), Sevcsik et al. reported that their interaction with the human multifunctional peptide LL-37 shows that net charge of the lipid is not the decisive factor determining the membraneperturbing mechanism of LL-37. The perturbation is induced by a combination of several parameters, including the packing density, the ability to form intermolecular H-bonds, and the molecular shape of the lipid..$^{79}$ 
The organisation of the PTH molecules around a phospholipid impurity centre would also result in a uniformly charged annulus (where the actual charge depends upon the individual PTH molecule in question and the local $\mathrm{pH}$ ). Without counter ions, this would be expected to induce a destabilising force between the individual PTH molecules, but it may also be this force repulsion that together with hydrophobic attractive forces between the ring structures and the acyl chains, helps push the PTH molecules deeper into the lipid bilayer in each successive turn of the helix.

The PTH and phospholipid molecule 'complex' will present a fairly large structure (extending up to approximately $60 \AA$ from Table 3). Such a structure would also be expected to have a significant effect on the local membrane curvature strain, surface charge, fluidity, and melting point. This could be the basis for the observations that trifluoperazine forms drug-rich domains in membranes, which in turn lead to the inhibition of the outward transport of anticancer drugs by the glycoprotein $\mathrm{P}$ (Pgp) in cancer cells. ${ }^{\mathbf{5}, 80}$ Whether the changes in membrane properties are the result of incomplete miscibility, as suggested by Cieslik-Boczula et al., or whether it is due to the molecular organisation suggested here, is yet to be determined.

Depression of the membrane's melting point by anaesthetic molecules has been known for a long time. Recently, Græsbøll et al., described a general model to explain the anaesthetic features of arbitrary molecules, in which they proposed that 'anaesthetics are ideally soluble in the liquid phase of the membrane only and are insoluble in the solid phase. ${ }^{81}$ Such a PTH and phospholipid molecule complex as described here would clearly have significantly different entropies in the liquid and solid states due to the 'accessibility' of individual phospholipid molecules.

\section{Experimental}

\section{Gravimetric micro-titration}

We used the simplest preliminary method, gravimetric microtitration, to examine the behaviour of the PTH/PL interactions in water to avoid possible buffer or ion-adjusting salts effects, particularly in view of the fact that PTH behaves as buffers in microclimates. In addition, we confined ourselves to PTH in hydrochloride form to avoid anionic differences. ${ }^{52}$

\section{Reagents}

The reagents were used as received. The PTH $\cdot$ hydrochlorides (Fig. 1A) came from Sigma Chemical Co., and all were infinitely water-soluble for our experimental purpose. Avanti Polar Lipids supplied the natural product extracts, as well as all the purified (99\% or better), synthetic 1, 2-diacyl-sn-glycero-3 -phospholipids (PL) with different headgroups (HG), and with even-numbered, symmetric, saturated diacyl chains varying generally from 8 to 24 carbon atoms per chain. Such numbers are indicated variously as iC or $\mathrm{Ci}$, e.g., 16PL or C16. The PL headgroups were choline (PC), ethanolamine (PE), the mono-sodium salts of phosphate (PA), rac-1-glycerol (PG), and L-serine (PS). The other PL were 16 -methyl-substituted PE (16PE Me, 16PE 2Me). The natural product extracts were Eschericia coli B, egg- and brain-sphingomyelin, (ESM) and (BSM) respectively, and phosphatidyl inositol
(PI). The PL molar weights may be found in the Avanti Products Catalogue Revised VI (homepage, http://www.avantilipids.com), which also gives extract compositions used here.

\section{Equipment}

Denver A200DS balance $\left(10^{-5} \mathrm{~g}\right)$, Oakton $1000 \mathrm{pH}$ meter (0.01), (6 mm) Accumet pH electrode, Haake circulating pumps, ColeParmer 8848 ultrasonic bath, silanized microsyringes, $15 \mathrm{~mL}$ silanized glass vials with Bakelite screw-caps, the latter never in contact with mixtures and solutions. The silanization product was Dow Coming MDX4-4159 Fluid provided by Factor II, Inc. P.O. Box 1339, Lakeside, Arizona 85929. The vials were silanized to obviate the negative surface charge on glass, also found on membranes and vesicular surfaces that could affect the course of PTH/PL aggregation. ${ }^{20,35}$

\section{Procedure}

Water was added to accurately weighed PL powder (in the range of 1-3 mg) yielding ultimately $\sim 10^{-5} \mathrm{M}$, (i.e., within an order of magnitude of the $10^{-6} \mathrm{M}$ critical micelle concentration (CMC) of biological PL, most of which have two long acyl chains ${ }^{17}$ ). Moreover, the PL were fully hydrated (see later). The suspension was subjected to sonication and heating to several degrees Celsius beyond the corresponding $\mathrm{PL}$ phase transition temperatures; ${ }^{82}$ sonicated again at $37{ }^{\circ} \mathrm{C}$; cooled to $0{ }^{\circ} \mathrm{C}$; again sonicated at $37{ }^{\circ} \mathrm{C}$; and then incubated at $37^{\circ} \mathrm{C}$, (each step for 1-3 min). The PTH solution ( 0.15 M) was added by a microsyringe in weighed increments, the mixture was subjected to the above temperature cycle, and addition and cycling were repeated until the turbidity cleared, which was taken to be the adduct endpoint and expressed as the PTH/PL molar ratio (MR). The solution was clear in a temperature range of several degrees Celsius on either side of the MR. After each incubation, the vial and contents were allowed to reach room temperature, upon which the vial (externally) and cap (internally and externally) were dried with lintless tissue, and the assembly tared for further additions of PTH. At room temperature and MR, the $\mathrm{pH}$ of the adduct solutions was compared with that of the equivalent pure PTH concentration for relatively soluble PL with small iCnumbers (see Avanti Catalog). After the MR had been reached and the $\mathrm{pH}$ taken, the PTH/PL mixtures were stored in the dark for evaluation of colour production, and possible reprecipitation.

\section{Conclusions}

We have presented data to illustrate that the interaction products of PTH and PL are water-soluble and stoichiometric, their molar ratio (MR) following a defined order of the headgroups (Table 1). Within this group, phosphatidyl ethanolamine-mono-methyl (PENMe) and phosphatidyl ethanolamine-di-methyl (PE 2Me) must be counted. The MR in vivo interaction products $\mathrm{PTH} / \mathrm{PL}$ are always smaller than the PTH/precursor PL. The increase in hydrogen ion concentration $\left[\Delta_{\mathrm{i}} \mathrm{H}^{+}\right]$is always larger for the $\mathrm{PTH} / \mathrm{PL}$ than for the equivalent pure PTH concentration $\left[\Delta_{\mathrm{i}} \mathrm{H}^{+}\right]$, and it is strongly dependent on the diacyl chain length. Each chain length in Fig. 3 contains all the PTH tested in the abovementioned order. 
This confirms Yeagle's claim of the partial independence of the headgroup and the acyl groups of a PL. ${ }^{43}$

Finally, the reproducibility of the molar ratio suggests that PTZ and PL interact in a defined matrix as adducts and not as loose aggregates. Because the results for all the various PTZ always retained the order of Series 2, this suggests that the adducts formed are similar in all the cases. Therefore, we used the molar ratios and knowledge about the forces between the molecules to propose that a phospholipid can act like an impurity centre, around which PTZ can form a helix. If the chain length is sufficiently long and the headgroup is small, the two lipid chains can splay to maximise the hydrophobic binding. The correlation between the molar ratio and the Fibonacci series suggests that the adducts formed are well organised to minimise the energy relationships.

A comparison of the data obtained here with the known therapeutic effects of these derivatives leads to the observation that the therapeutic phenothiazine derivatives (PTH) can be divided into two overlapping groups. One of these (referred to here as Series 1) is psychotropic and based on heterocyclic ionization potentials. The other (Series 2) is based on lipophilicity and is related to the non-neuronal effects on cellular membrane phospholipids, which results in their therapeutic effectiveness against deleterious micro-organisms and cancer cells.

\section{Abbreviations}

$\begin{array}{ll}\text { CL } & \text { Chain length } \\ I_{\mathrm{p}} & \text { Ionization potential } \\ \text { MR } & \text { Molar ratio } \\ \text { PL } & \text { Phospholipid } \\ \text { CPZ } & \text { Chlorpromazine } \\ \text { PClP } \cdot \mathrm{HCl} & \text { Prochlorperazine } \cdot \mathrm{HCl} \\ \text { PClP } \cdot 2 \mathrm{HCl} & \text { Prochlorperazine } \cdot \text { 2HC1 } \\ \text { PMTZ } & \text { Promethazine } \\ \text { PMZ } & \text { Promazine } \\ \text { PTH } & \text { Phenothiazine hydrochloride derivatives } \\ \text { TFM } & \text { Triflupromazine } \\ \text { TFP } \cdot \mathrm{HCl} & \text { Trifluoperazine } \cdot \mathrm{HCl} \\ \text { TFP } \cdot 2 \mathrm{HCl} & \text { Trifluoperazine } \cdot 2 \mathrm{HCl} \\ \text { PA } & \text { Phosphatidate (or phosphatidic acid) } \\ \text { PC } & \text { Phosphatidylcholine } \\ \text { PE } & \text { Phosphatidylethanolamine } \\ \text { PENMe } & \text { PE16Nmethyl } \\ \text { PENMe2 } & \text { PE16Ndimethyl } \\ \text { PG } & \text { Phosphatidyl glycerol } \\ \text { PS } & \text { Phosphatidylserine } \\ \text { CaL } & \text { Cardiolipin }\end{array}$

\section{Acknowledgements}

We thank Elysia Thornton-Benko for her support and medical information on micelles and stability during the early period of the project, and we thank Vivian C. Flores for her assistance in completing the manuscript.

\section{References}

1 J. E. Kristiansen, O. Hendricks, T. Delvin, T. S. Butterworth, L. Aagaard, J. B. Christensen, V. C. Flores and H. Keyzer, J. Antimicrob. Chemother., 2007, 59, 1271-1279.

2 G. Spengler, J. Molnar, M. Viveiros and L. Amaral, Anticancer Res., 2011, 31, 4201-4205.

3 M. J. Ohlow and B. Moosmann, Drug Discovery Today, 2011, 16, 119-131.

4 A. Jaszczyszyn, K. Gasiorowski, P. Swiatek, W. Malinka, K. Cieslik-Boczula, J. Petrus and B. Czarnik-Matusewicz, Pharmacol. Rep., 2012, 64, 16-23.

5 G. Spengler, D. Takacs, A. Horvath, Z. Riedl, G. Hajos, L. Amaral and J. Molnar, Anticancer Res., 2014, 34, 17371741.

6 D. Zong, K. Zielinska-Chomej, T. Juntti, B. Mork, R. Lewensohn, P. Haag and K. Viktorsson, Cell Death Dis., 2014, 5, e1111.

7 D. Takacs, P. Cerca, A. Martins, Z. Riedl, G. Hajos, J. Molnar, M. Viveiros, I. Couto and L. Amaral, In Vivo, 2011, 25, 719724.

8 S. Sharma and A. Singh, Expet. Opin. Investig. Drugs, 2011, 20, 1665-1676.

9 P. K. Dea, H. Keyzer and J. S. Maurer, in Phenothiazines and 1,4-Benzothiazines, ed. R. R. Gupta, Elsevier, New York, 1988, pp. 587-625.

10 P. M. Seeman, Pharmacol. Rev., 1972, 24, 583-655.

11 J. Hyttel, J. Arnt and K. P. Bøgesø, Antipsychotic drugs: Configurational stereoisomers, CRC Press, Boca Raton, Florida, 1984.

12 J. Schmutz and C. W. Picard, in Psychotropic Agents. Part I: Antipsychotics and Antidepressants, ed. F. Hoffmeister and G. Stille, Springer, 1980, vol. 55(1), pp. 3-26.

13 S. J. Fey and K. Wrzesinski, Toxicological Sciences : an Official Journal of the Society of Toxicology, 2012, 127, 403-411.

14 M. J. Allen, Electrochim. Acta, 1966, 11, 1503-1504.

15 M. J. Allen, Electrochim. Acta, 1967, 12, 563-568.

16 J.-C. Pechere, Abstracts of the Seventh European Congress of Chemotherapy and Infection, Florence, Italy, 2005, 2005, Abstract EL 1, pp. 19-22.

17 M. W. Goosey and N. S. Doggett, Biochem. Pharmacol., 1983, 32, 2411-2416.

18 F. Gutmann, C. Johnson, H. Keyzer and J. Molnar, Charge Transfer Complexes in Biological Systems, Marcel Dekker, New York, 1997.

19 K. Michalak, O. Wesolowska, N. Motohashi, J. Molnar and A. B. Hendrich, Curr. Drug Targets, 2006, 7, 1095-1105.

20 M. R. Fernandez, H. Keyzer, M. Dea and P. K. Dea, in Thiazines and Structurally Related Compounds. Proceedings of the Sixth International Conference on Phenothiazines and Structurally Related Psychotropic Compounds, sept 1114, 1990, ed. H. Keyzer, G. M. Eckert, I. S. Forrest and R. R. Gupta, Krieger Publishing Company, Pasadena, 1992, pp. 187-191.

21 A. I. McMullen and J. A. Stirrup, Biochim. Biophys. Acta, 1971, 241, 807-814. 
22 P. Maher and S. J. Singer, Biochemistry, 1984, 23, 232-240.

23 C. D. Francesco and M. H. Bickel, Chem.-Biol. Interact., 1977, 16, 335-346.

24 M. Ahmed, J. Hadgraft and I. W. Kellaway, Int. J. Pharmacol., 1983, 13, 227-237.

25 R. M. Julien, A primer of drug action, W.H. Freeman, San Francisco, 1975.

26 H. Keyzer, H. K. Kim and C. Varkey-Johnson, in Thiazines and Structurally Related Compounds, ed. H. Keyzer, G. M. Eckert, I. S. Forrest and R. R. Gupta, Krieger Publishing company, Florida, 1992, pp. 213-218.

27 M. C. Carey, P. C. Hirom and D. M. Small, Biochem. J., 1976, 153, 519-531.

28 V. C. Flores, H. Keyzer, H. K. Kim and J. Molnar, Anticancer Res., 2002, 22, 959-967.

29 R. Welti, L. J. Mullikin, T. Yoshimura and G. M. Helmkamp Jr, Biochemistry, 1984, 23, 6086-6091.

30 R. L. Smith and E. Oldfield, Science, 1984, 225, 280-288.

31 S. Tehrani, N. Brandstater, Y. D. Saito and P. Dea, Biophys. Chem., 2001, 94, 87-96.

32 L. R. Barbosa, R. Itri, W. Caetano, S. Neto Dde and M. Tabak, J. Phys. Chem. B, 2008, 112, 4261-4269.

33 P. Mukerjee and A. K. Ghosh, J. Am. Chem. Soc., 1970, 92, 6403-6407.

34 R. J. Abraham, L. J. Kricka and A. Ledwith, J. Chem. Soc., Chem. Commun., 1973, 8, 282-283.

35 V. Flores, C. Varkey-Johnson and H. Keyzer, in Thiazines and Structurally Related Compounds of Biological Significance, ed. J. Barbe, Enlight Assoc., San Gabriel, USA, 1994.

36 B. S. Thornton, Phys. Lett. A, 1984, 106, 198-202.

37 J. Molnar, B. S. Thornton, E. Thornton-Benko, L. Amaral, S. Zsujsanna and M. Novak, Curr. Cancer Ther. Rev., 2009, 5, 158-169.

38 H. Hauser and G. Poupart, in The Structure of Biological membranes, ed. P. Yeagle, CRC Press, Boca Raton, 1992, pp. 3-72.

39 D. Voet and J. G. Voet, Biochemistry, Wiley and Son, New York, 2nd edn, 1995.

40 L. Vroman, in Interfacial Phenomena in Biological Systems, ed. M. Bender, Marcel Decker, New York, 1991.

41 J. R. Silvius, Lipid-Protein Interactions, Wiley and Son, New York, 1982.

42 M. J. Mercier and P. A. Dupont, J. Pharm. Pharmacol., 1972, 24, 706-712.

43 P. Yeagle, in The Structure of Biological Membranes, ed. P. Yeagle, CRC press, Boca Raton, 1997, pp. 157-174.

44 R. Knoesel, B. Gebus, J. P. Roth and J. Parrod, Bull. Soc. Chim. Fr., 1969, 1, 294-301.

45 B. Gebus, R. Knoesel and J. Parrod, Bull. Soc. Chim. Fr., 1969, 1, 290-294.

46 J. E. Bloor, B. R. Gilson, R. J. Haas and C. L. Zirkle, J. Med. Chem., 1970, 13, 922-925.

47 A. Fulton and L. E. Lyons, Aust. J. Chem., 1968, 21, 873-882. 48 S. H. Snyder and E. Richelson, Proc. Natl. Acad. Sci. U. S. A., 1968, 60, 206-213.
49 Q. Duncan, H. Keyzer and K. L. Young, in Biological and Chemical Aspects of Thiazines and Analogues, ed. J. Barbe, H. Keyzer and J. C. Soyfer, Enlight Association, San Gabriel, California, 1995, pp. 131-140.

50 G. L. Jendrasiak and J. C. Mendible, Biochim. Biophys. Acta, 1976, 424, 149-158.

51 R. P. Rand and V. A. Parsegian, in The Structure of Biological Membranes, ed. P. Yeagle, CRC press, Boca Raton, 1977.

52 P. K. Dea and H. Keyzer, Conformational and Electronic Aspects of Chlorpromazine in Solution, Plenum Press, 1986.

53 S. M. Gruner, M. W. Tate, G. L. Kirk, P. T. So, D. C. Turner, D. T. Keane, C. P. Tilcock and P. R. Cullis, Biochemistry, 1988, 27, 2853-2866.

54 S. Mulukutla and G. G. Shipley, Biochemistry, 1984, 23, 25142519.

55 A. T. Florence, Adv. Colloid Interface Sci., 1968, 2, 115-149.

56 A. T. Florence, J. Pharm. Pharmacol., 1970, 22, 1-9.

57 D. J. Vaughan and K. M. Keough, FEBS Lett., 1974, 47, 158161.

58 A. T. Florence, in Micellisation, Solubilisation and Microemulsions, ed. K. L. Mittal, Plenum Press, New York, 1977, vol. 1, pp. 50-74.

59 B. Bondy, Gen. Pharmacol., 1988, 17, 261-265.

60 V. Flores, H. Keyzer and J. E. Kristiansen, in 6th European Congress on Chemotherapy and Infection (Dec. 1-3), Paris, France, 2004, p. Abstract 669.

61 M. Almgren, Biochim. Biophys. Acta, 2000, 1508, 146-163.

62 A. Zachowski and P. Durand, Biochim. Biophys. Acta, 1988, 937, 411-416.

63 L. Zhao and S. S. Feng, J. Colloid Interface Sci., 2004, 274, 5568.

64 L. Zhao, S. S. Feng and M. L. Go, Eur. J. Pharm. Sci., 2004, 93, 86-98.

65 L. Zhao and S. S. Feng, J. Colloid Interface Sci., 2005, 285, 326-335.

66 W. Kopec and H. Khandelia, J. Comput.-Aided Mol. Des., 2014, 28, 123-134.

67 T. Rog, M. Pasenkiewicz-Gierula, I. Vattulainen and M. Karttunen, Biochim. Biophys. Acta, 2009, 1788, 97-121.

68 G. W. Grossman, Applications of Fibonacci numbers, 1997, 9, 211-224.

69 G. M. Carman and G. S. Han, J. Lipid Res., 2009, 50, S69S73.

70 A. Nohturfft and S. C. Zhang, Annu. Rev. Cell Dev. Biol., 2009, 25, 539-566.

71 A. M. Cardozo Gizzi and B. L. Caputto, IUBMB Life, 2013, 65, 584-592.

72 A. Shevchenko and K. Simons, Nat. Rev. Mol. Cell Biol., 2010, 11, 593-598.

73 C. Bissig and J. Gruenberg, Cold Spring Harbor Perspect. Biol., 2013, 5, a016816.

74 G. van Meer, D. R. Voelker and G. W. Feigenson, Nat. Rev. Mol. Cell Biol., 2008, 9, 112-124.

75 N. D. Ridgway, Crit. Rev. Biochem. Mol. Biol., 2013, 48, 20-38. 76 S. Piotto, A. Trapani, E. Bianchino, M. Ibarguren, D. J. Lopez, X. Busquets and S. Concilio, Biochim. Biophys. Acta, 2014, 1838, 1509-1517. 
77 V. Teixeira, M. J. Feio and M. Bastos, Prog. Lipid Res., 2012, 51, 149-177.

78 B. Bechinger, Mol. Membr. Biol., 2000, 17, 135-142.

79 E. Sevcsik, G. Pabst, W. Richter, S. Danner, H. Amenitsch and K. Lohner, Biophys. J., 2008, 94, 4688-4699.
80 K. Cieslik-Boczula, P. Swiatek, A. Jaszczyszyn, P. Zawilska, K. Gasiorowski, W. Malinka and G. Kohler, J. Phys. Chem. $B, 2014$, 118, 3605-3615.

81 K. Graesboll, H. Sasse-Middelhoff and T. Heimburg, Biophys. J., 2014, 106, 2143-2156.

82 A. Harder and H. Debuch, Chem. Phys. Lipids, 1986, 39, 6571. 